\title{
Accuracy of corneal trephination depth using the Moria single-use adjustable depth vacuum trephine system
}

This article was published in the following Dove Press journal:

Clinical Ophthalmology

27 November 2014

Number of times this article has been viewed

\author{
Carlton R Fenzl' \\ Adam J Gess ${ }^{2}$ \\ Majid Moshirfar ${ }^{3}$ \\ 'John A Moran Eye Center, University of \\ Utah, Salt Lake City, UT, ${ }^{2}$ Eye Doctors of \\ Washington, Washington, DC, ${ }^{3}$ Cornea \\ and Refractive Surgery Division, \\ Department of Ophthalmology, Francis \\ I Proctor Foundation, University of \\ California San Francisco, San Francisco, \\ CA, USA
}

Background: The Moria single-use adjustable depth trephine is a device that allows a goal trephination depth to be set prior to the surgical procedure.

Methods: Eleven fresh human cadaveric eyes were trephined using $8.0 \mathrm{~mm}$ Moria singleuse adjustable vacuum trephines. Prior to trephination, the average corneal pachymetry in the peripheral 7-10 $\mathrm{mm}$ range was obtained using anterior segment optical coherence tomography. The trephination depth was set to $80 \%$ of that value. Light microscopy was used to image anteroposterior cross-sections of each corneal specimen. Digital protractor software was used to evaluate the trephination angle, depth, and length. All adequately processed specimens were included in the analysis. In addition, trephination angle data from a previous publication by Moshirfar et al were used as a comparison with those of this study.

Results: Trephination analysis of depth compared with pachymetry revealed a mean of $83.7 \% \pm 6.53 \%$ (95\% confidence interval 79.8-87.6). Maximum and minimum trephined depths were $95.35 \%$ and $71.3 \%$, respectively. Trephination depth compared with angular corneal thickness yielded a mean of $66.2 \% \pm 4.79 \%$ (95\% confidence interval 63.0-69.4). Maximum and minimum depths were $73.7 \%$ and $59.7 \%$, respectively. Analysis of trephination angle yielded a mean of $130.2 \pm 3.57$ degrees (95\% confidence interval 127.8-132.61). Maximum and minimum angles were 135.5 degrees and 126 degrees, respectively. The standard deviation of the trephination angle of the Moria trephine was found to be significantly less than that of Hessburg-Barron and Hanna trephines calculated in the previous study.

Conclusion: The Moria adjustable vacuum trephine is an accurate method of trephination when a specific depth is desired. Further investigation is needed to determine the relevance of this in relation to deep anterior lamellar keratoplasty.

Keywords: trephine, Moria, deep anterior lamellar keratoplasty, adjustable depth, vacuum

\section{Introduction}

The Moria (Moria Surgical, Antony, France) single-use adjustable depth trephine is a new device that combines the advantages of a vacuum-based anteriorly performed system with an additional benefit, ie, an adjustable depth meter (Figure 1). ${ }^{1-3}$ The creation of precise trephination incisions is vital in several surgical procedures. The most important of these is deep anterior lamellar keratoplasty (DALK), which requires removal of the corneal epithelium, Bowman's membrane, and stroma down to the deep layers. ${ }^{4}$ The use of a corneal trephine to help with this process is necessary in most variations of DALK. An appropriately performed trephination helps the surgeon establish a cleavage plane that ideally separates Descemet's membrane from stroma. ${ }^{5-7}$ However, the goal depth of trephination is not consistent amongst authors, and a range is often given. Examples include 60\%-80\% depth and 300-400 $\mu \mathrm{m} .{ }^{4,5}$ Some surgeons 


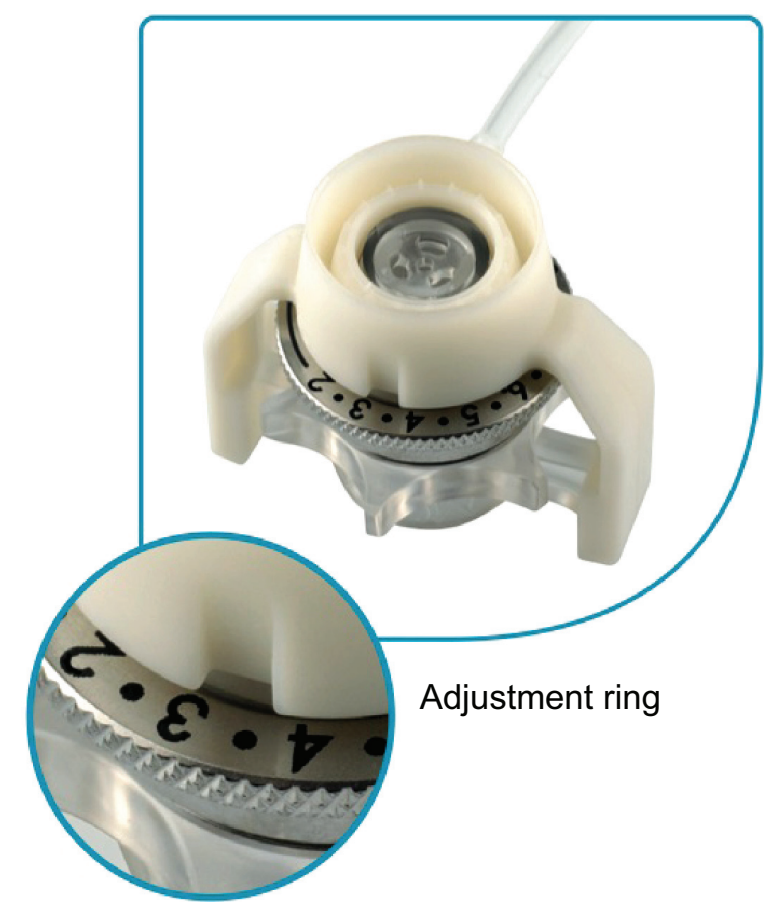

Figure I Photograph of the adjustable depth meter present on the Moria single-use adjustable vacuum trephine.

estimate the depth by counting blade rotations. This likely results in significant variability. Anwar and Teichmann stressed the importance of using a calibrated trephine to increase accuracy. ${ }^{5}$

This paper addresses a new adjustable corneal trephine, which proposes to more accurately standardize trephination depth, angulation, and centration. Proposed advantages of this new trephine include central corneal flattening for straighter cuts and an adjustable depth meter that is labeled in $50 \mu \mathrm{m}$ increments, but can be calibrated to any depth desired. This study aims to evaluate these claims on fresh human cadaveric eyes, using anterior segment optical coherence tomography (AS-OCT; Visante, Zeiss, Jena, Germany) and histopathologic evaluation of depth and angulation.

\section{Materials and methods}

Eleven fresh human cadaveric eyes were trephined using $8.0 \mathrm{~mm}$ Moria single-use adjustable vacuum trephines. Tissue was donated for research purposes from the Utah Lions Eye Bank, Salt Lake City, UT, USA. Each specimen was deemed unsuitable for transplantation because of failure of serologic screening. Using a 27-gauge needle and a 5 cc syringe, Balanced Saline Solution (Alcon, Fort Worth, TX, USA) was injected into the vitreous cavity to obtain an intraocular pressure of $40 \pm 3 \mathrm{mmHg}$. The globes were then stabilized on a metallic base, and AS-OCT was performed. Using the average corneal pachymetry in the peripheral $7-10 \mathrm{~mm}$ range (Figure 2), as derived from the AS-OCT, the trephine depth was adjusted to $80 \%$. For example, for a 1,000 micron cornea, the trephine was set at 800 microns. The trephine was placed centrally on the cornea and proper vacuum suction was confirmed. Trephination proceeded until a hard stop was felt. Each globe was trephined by the same operator using a new trephine for each eye. An incision was then made $1 \mathrm{~mm}$ posterior to the limbus, and curved scissors were used to cut circumferentially leaving the cornea with a small scleral rim. The tissues were processed using standard histopathologic processing techniques. Corneoscleral buttons were cut in an anteroposterior manner in eight equally spaced meridians, and light microscopy was performed on each section.

Data were obtained using measurements taken from light microscopic specimens. Digital protractor software (AutoCAD 2013, Autodesk, San Rafael, CA, USA) was used to evaluate the trephination angle, depth, and length. Trephination depth was determined to be the length of the incision measured from the start to the completion of the trephination. Digital straight line segments with protractor capabilities were placed at the epithelial origin of the trephination incision and extended to the aforementioned points within the stroma. Corneal thickness was calculated in a similar manner yielding two distinct measurements. Pachymetry (Figure 3) was determined to be the distance from epithelium to endothelium oriented at an angle 90 degrees to the epithelial surface. Angular corneal thickness (ACT) (Figure 4) was deemed to be the distance from epithelium to endothelium at an angle parallel to the trephination incision. Trephination angle was calculated in a similar manner (Figure 5).

Depth was then calculated as a percentage of each respective corneal thickness rather than an absolute value (Figures 3 and 4). This was done to account for tissue shrinkage that occurs during tissue processing. In no case were all eight sections in proper condition for study inclusion. Inadequate sections (eg, folded or damaged tissue) were excluded from analysis. All adequate cross-sections were included, those from the same sample were averaged, and numerical means were obtained. Standard deviations and 95\% confidence intervals (CIs) were calculated to evaluate the accuracy of the trephine.

Additionally, raw data were obtained from a previous study performed by Moshirfar et al (Figure 6). Trephination incision angles obtained using Hessburg-Barron (Katena 


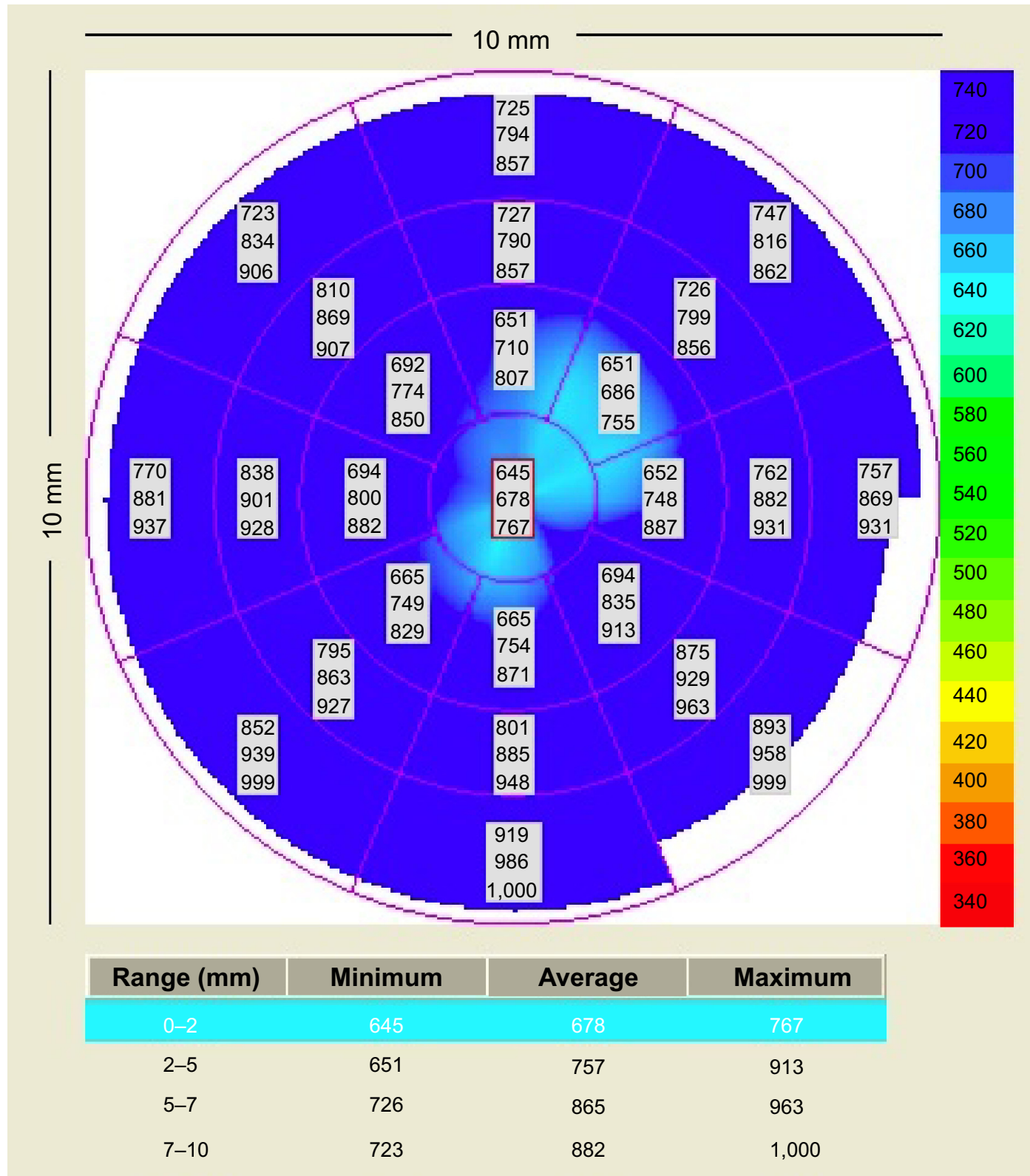

Figure 2 Example of corneal pachymetry using anterior segment optical coherence tomography. An average range of 7-10 mm was used.

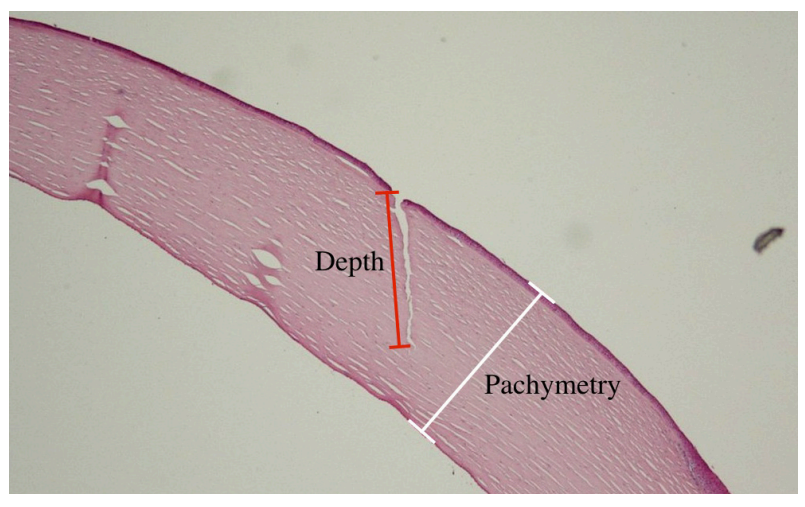

Figure 3 Light microscopic corneal specimen demonstrating incision depth and pachymetry.

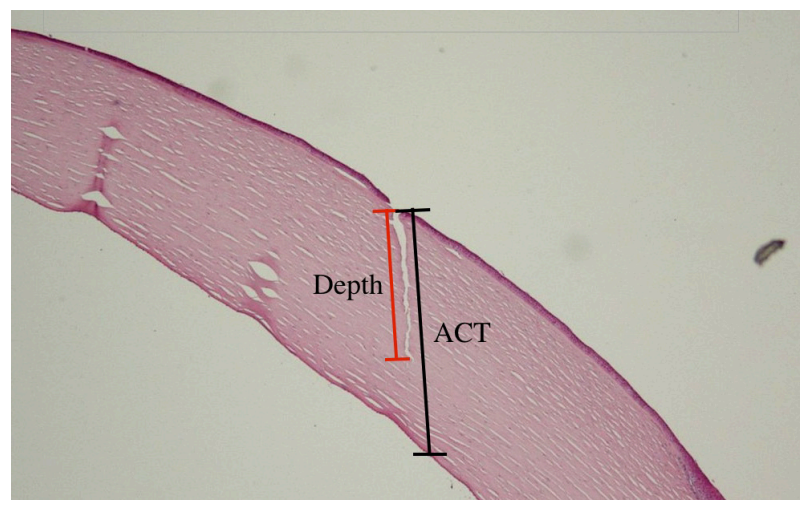

Figure 4 Light microscopic corneal specimen demonstrating incision depth and ACT. Abbreviation: $\mathrm{ACT}$, angular corneal thickness. 


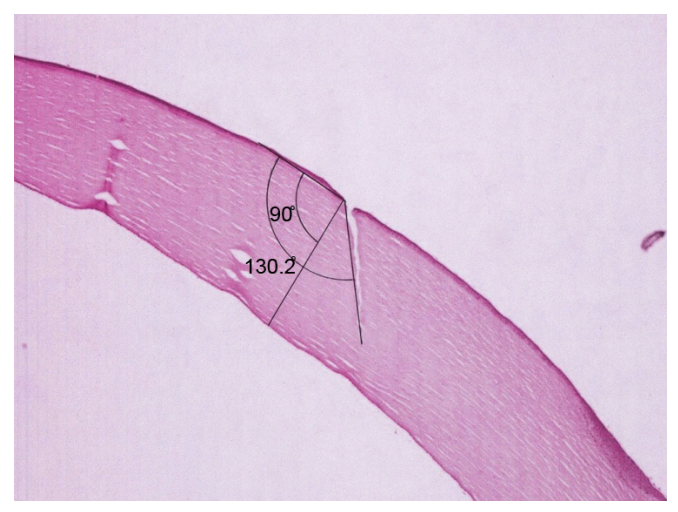

Figure $\mathbf{5}$ Light microscopic corneal specimen demonstrating incision angle in comparison with the epithelial surface.

Products Inc, Denville, NJ, USA) and Hanna (Moria Surgical) trephines were compared with those of the Moria trephine using means and standard deviations. In the previous study, the protocol for trephination was identical to that of this one except for the type of trephine used and the fact that a full thickness corneal incision was produced. ${ }^{8}$

\section{Results}

Trephination was successful in all eyes, with no episodes of suction loss. In no cases did the trephine penetrate the anterior chamber. The mean of the average peripheral $7-10 \mathrm{~mm}$ corneal thickness values was $932.1 \pm 77.3$ microns. Histopathologic analysis of trephination depth compared with pachymetry (Figure 3) revealed a mean of $83.7 \% \pm 6.53 \%$ (95\% CI 79.8-87.6). Maximum and minimum trephined depths were $95.35 \%$ and $71.3 \%$, respectively (Figure 7 ). Trephination depth compared with ACT (Figure 4) yielded

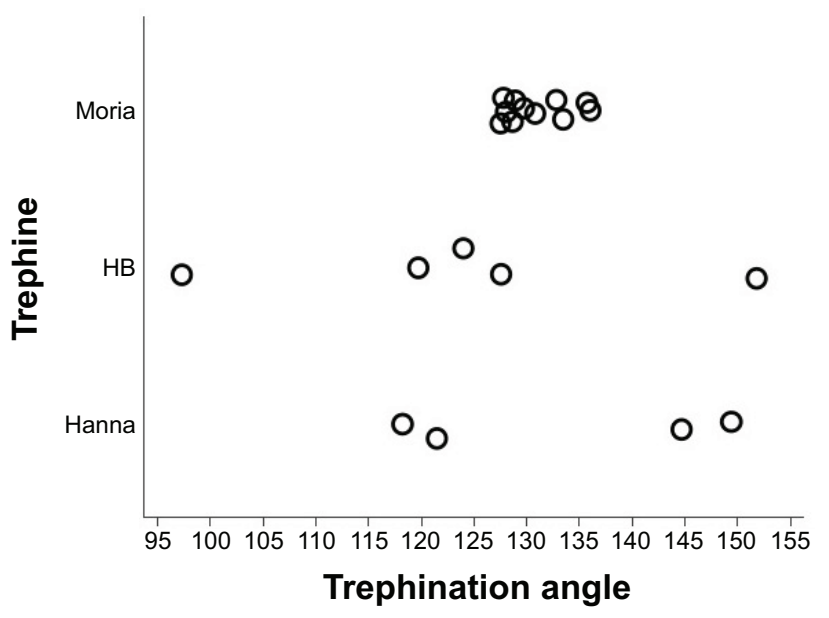

Figure 6 Comparison of the trephination angles (in degrees) of Moria*, HB, and Hanna trephines. *Moria single-use adjustable depth vacuum trephine. Abbreviation: $\mathrm{HB}$, Hessburg-Barron.

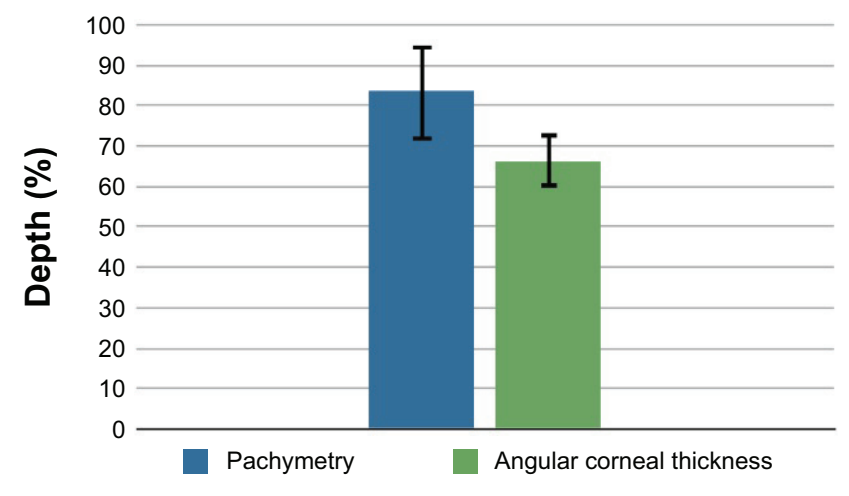

Figure $\mathbf{7}$ Graphic representation of the mean trephination depth as a percentage of corneal thickness of pachymetry versus that of angular corneal thickness. Ranges are indicated by black bars.

a mean of $66.2 \% \pm 4.79 \%$ (95\% CI $63.0-69.4)$. Maximum and minimum depths were $73.7 \%$ and $59.7 \%$, respectively (Figure 7). Analysis of trephination angle yielded a mean of 130.2 23.57 degrees (95\% CI 127.8-132.61). Maximum and minimum angles were 135.5 degrees and 126 degrees, respectively.

In our previous study, analysis of the trephination angle for the Hanna and Hessburg-Barron corneal trephines demonstrated $95 \%$ CIs of $111.9-157.7$ and 101.9-150.2, respectively. ${ }^{8}$ Figure 6 provides a graphical representation of the three data sets. Two-sample $t$-tests with unequal variances showed no significant differences in the trephination angle data sets. However, the standard deviation of the Moria trephination angles was found to be significantly less than both the Hessburg-Barron $(P<0.05)$ and Hanna $(P<0.05)$ trephination angles using the Levene's test of equal variances.

\section{Discussion}

The Moria adjustable-depth trephine is an anteriorly performed, vacuum-based system that has the ability to select a goal depth at which trephination is halted. This allows for increased precision when performing DALK and penetrating keratoplasty, when the surgeon wants to stop short of a complete trephination.

This study was designed to evaluate the accuracy of this trephination device both in terms of the depth selector and the incision angle. Preprocedural pachymetry measured by AS-OCT (white bracket, Figure 3) was designated as the thickness against which the trephination depths (red bracket, Figure 3) were to be compared. Mean incision depth was determined to be $83.7 \%$. These results indicate that the average trephination depth is accurate within $4 \%$ points. 
There is, however, a major flaw in using imaging modalities such as AS-OCT preprocedurally to calculate corneal thickness. AS-OCT is limited in its ability to obtain anything other than perpendicular measurements of corneal thickness as the basis for depth calculation. ${ }^{9}$ Due to the prolate nature of the cornea and the peripheral location at which the trephination blade rests, trephination proceeds at an angle not directly perpendicular to the surface of the cornea. As a result, the blade must pass through a greater amount of corneal tissue before penetration into the anterior chamber than was originally estimated by the AS-OCT. This results in an underestimation of incision depth.

Figure 4 illustrates the angular nature of both the trephination incision (red brackets) and the ACT (black brackets). A comparison of these two data sets yields a mean depth of $66.2 \%$. As expected, the percentage depth is less (17.5\%) than the comparison between incision depth and pachymetry. This error stems from the inappropriate selection of pachymetry for use in depth calculations. Had ACT been used preprocedurally, a mean incision depth of approximately $83.7 \%$ would have been observed.

$\mathrm{ACT}$ can be quickly and easily obtained through use of a conversion factor. In this study, a conversion factor of 1.175 was calculated by taking the difference between the average ACT and pachymetry. Take, for example, a patient with an average peripheral pachymetry of $750 \mu \mathrm{m}$. Conversion using the conversion factor of 1.175 yields an ACT of $881.25 \mu \mathrm{m}$. The goal depth of trephination can then be calculated from this number. If the study goal of $80 \%$ is used, $705 \mu \mathrm{m}$ should set using the depth meter. This conversion factor is reserved specifically for the $8.0 \mathrm{~mm}$ diameter trephine. Due to changes in angulation and thickness of the peripheral cornea, separate conversion factors should be calculated for all sizes and brands of trephine in use. Failure to convert will result in consistently shallow trephination incisions.

Finally, the narrow range of angles produced by the Moria trephine is important because it indirectly affects the depth penetration. This is illustrated by the significant difference in incision angle standard deviation compared with the two other trephines. If the microns of cornea through which the trephination blade can penetrate is kept constant, an incision created at an angle of 100 degrees to the corneal epithelium is much closer to penetration into the anterior chamber than one created at 150 degrees. As a result, a consistent incision angle is a vital step in creating accurate incision depth.

Limitations of this study include the relatively small sample size and the possible effects of histopathologic processing on tissue thickness and angle measurements. While the tissue thickness is undoubtedly affected by histopathologic processing, all corneal tissue would be expected to shrink symmetrically, leaving the relative percentages unchanged. Our prior study used AS-OCT to measure post-trephination thickness; however, these corneas were trephined completely. As this was a partial-thickness trephination, shadowing prevented accurate measurements of post-trephine depth. This resulted in the requirement for histopathologic analysis of depth and trephine angle.

\section{Conclusion}

This study suggests that the Moria adjustable-depth corneal trephine is an accurate method for trephination to a specific corneal depth, with a mean depth of only slightly greater than intended. In addition, the standard deviation of trephination angles of the Moria trephine was significantly less than those observed with the Hessburg-Barron and Hanna trephines. This suggests a difference in consistency of clinical results. It is vital that surgeons be diligent about performing depth calculations using ACT. Imaging modalities measuring pachymetry may be used as long as standardized or personalized formulas are used to convert to ACT.

A great fear amongst surgeons performing DALK is the penetration of Descemet's membrane. This is likely the reason for recommendation of a broad range of trephination depths. A deeper, more consistent trephination depth may be what is necessary to achieve the appropriate split between Descemet's membrane and stroma for the great majority of patients. Further investigation is needed in determining the relevance of trephination depth in DALK.

\section{Disclosure}

The authors report no conflicts of interest in this work. No funding was provided by Moria Surgical.

\section{References}

1. Jacobsen N, Hojgaard-Olsen K. Promising results using the Hanna corneal trephine system in penetrating keratoplasty. Cornea. 2006;25: 371-376.

2. Radner W, Skorpik C, Loewe R, Mudrich C, Radner G, Mallinger R. Effect of trephination technique on the ultrastructure of corneal transplants: guided trephine system v posterior punch technique. $\mathrm{Br} \mathrm{J} \mathrm{Oph-}$ thalmol. 1999;83:1172-1177.

3. Wilbanks GA, Cohen S, Chipman M, Rootman DS. Clinical outcomes following penetrating keratoplasty using the Barron-Hessburg and Hanna corneal trephination systems. Cornea. 1996;15:589-598.

4. Krachmer JH, Mannis MJ, Holland EJ. Cornea: Surgery of the Cornea and Conjunctiva. 3rd ed. New York, NY, USA: Elsevier Inc; 2011.

5. Anwar M, Teichmann KD. Big-bubble technique to bare Descemet's membrane in anterior lamellar keratoplasty. J Cataract Refract Surg. 2002; 28:398-403. 
6. Manche EE, Holland GN, Maloney RK. Deep lamellar keratoplasty using viscoelastic dissection. Arch Ophthalmol. 1999;117:1561-1565.

7. Sarnicola V, Toro P, Gentile D, Hannush SB. Descemetic DALK and predescemetic DALK: outcomes in 236 cases of keratoconus. Cornea. 2010;29:53-59.

8. Moshirfar M, Calvo CM, Kinard KI, Williams LB, Sikder S, Neuffer MC. Comparison of Hanna and Hessburg-Barron trephine and punch systems using histological, anterior segment optical coherence tomography and elliptical curve fitting models. Clin Ophthalmol. 2011;5: $1121-1125$.
9. Fante RJ, Shtein RM, Titus MS, Woodward MA. Anterior segment OCT versus ultrasound pachymetry to measure corneal thickness in endothelial keratoplasty donor corneas. Cornea. 2013;32(5):e79-82.
Clinical Ophthalmology

\section{Publish your work in this journal}

Clinical Ophthalmology is an international, peer-reviewed journal covering all subspecialties within ophthalmology. Key topics include: Optometry; Visual science; Pharmacology and drug therapy in eye diseases; Basic Sciences; Primary and Secondary eye care; Patient Safety and Quality of Care Improvements. This journal is indexed on

\footnotetext{
Submit your manuscript here: http://www.dovepress.com/clinical-ophthalmology-journal
}

\section{Dovepress}

PubMed Central and CAS, and is the official journal of The Society of Clinical Ophthalmology (SCO). The manuscript management system is completely online and includes a very quick and fair peer-review system, which is all easy to use. Visit http://www.dovepress.com/ testimonials.php to read real quotes from published authors. 